\title{
Breakthrough Discoveries vs Incremental Science
}

\author{
Didier Queloz, Mejd Alsari
}

Professor Didier Queloz talks about his challenging journey from the discovery of the first exoplanet orbiting a solar-type star in 1995 to the acknowledgement of his discovery by the scientific community. He explores his experience in reporting a paradigm-changing finding and how this triggered hard scepticism from the publishing industry and fellow scientists, which lasted about 3 years. He and Michel Mayor were eventually acknowledged as the founders of the new field of exoplanets and were awarded the Nobel Prize for Physics in 2019. See video at https://youtu.be/4cXalPDJT5k

MA. You said there were antecedents to your discovery and you mentioned this in your paper published in 1995. But you said that the field wasn't considered a serious topic. Was it because of resolution limits and false positives?

DQ. There is a long story in exoplanet science ${ }^{1,2}$ of false alarm or people claiming they found a planet and it was not a planet before we found the first one (orbiting a Sun-like star). ${ }^{3}$ Some people may have thought 'It's again the same kind of stuff, it isn't a planet!'

You have to realise that we setup the instrument and, one year after the first light, practically we had the first planet. So, it was extremely quick. Usually you have to establish some confidence with the machine. We were very confident but nobody was aware of this machine.

When the observations were confirmed by Geoffrey Marcy a couple of weeks later, ${ }^{4}$ people understood that the data was fine, but then came up the big debate: 'Can we explain the data?'

Maybe we just had a change in the radial velocity due to something other than a planet. We carefully studied that in the paper, but the topic was controversial. Some people suspected that maybe there were some effects, subtle effects, on the atmosphere of the 51 Pegasi star. They suggested an impossible precession (i.e. that the spectral-line variations were caused by a pulsating stellar surface), which is not observed on other stars, but that could apply to that one. ${ }^{5}$ That was ruled out in our paper, ${ }^{4}$ but I don't think they understood the way we did it. Then for some time, people were arguing whether this alternative explanation was right or not. ${ }^{6}$ Despite the fact that some people realised that the paper published in 1997 claiming that we did not detect a planet ${ }^{5}$ was wrong, ${ }^{7,8}$ the paper is still there.

At that time the community was still not very sure. Well, people really working on the topic, there were maybe 50 people in the world, were pretty sure that the planet was real, but the community outside the field did not really know what to think about that and they all knew that the planet was awkward. ${ }^{6}$

When you have a planetary theory and data that doesn't match this theory, usually the global physics attitude is being sceptical. If somebody tells you 'I made an experiment and I found that this particle goes faster than the speed of light,' you'll say 'Come on, this is a big theory'. If you do that, there are consequences.

In our case it was not that bad because I think formation theory is not like fundamental physics theory. But we had a nice picture of the solar system, and people were not really willing to trash it. 'It works for the solar system, so maybe there's something wrong', they must have thought.

It took some time to swallow.

What I learned later on is that the bigger the discovery the longer it takes for the discovery to be understood. If you're doing incre-

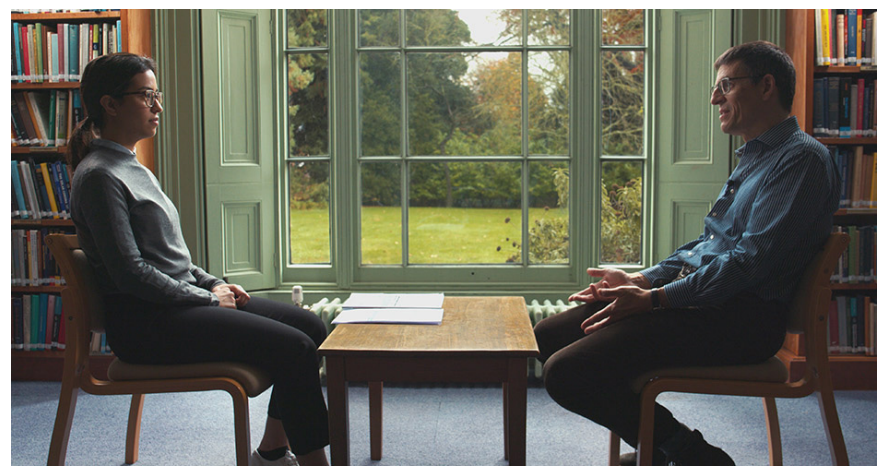

Figure 1 | Prof. Didier Queloz, Professor of Physics, Cavendish Laboratory, with Dr. Mejd Alsari, postdoctoral researcher, Cavendish Laboratory...

mental science usually it's fine. Everybody's expecting it, you're happy, you publish your paper, and everybody will be citing you. In our case, it was so awkward.

We were also trying to build up a theory. There were some attempts early on, but it was debated. New awkward planets kept coming. We had all these planets popping up but that weren't exactly the way we were expecting them to be. It took some time to get this right.

For me it was certainly something difficult because I was just a $\mathrm{PhD}$ student. In a way I wanted to move forward and do something else. I was a bit tired. There were people challenging what I did, other people telling me 'This is the greatest discovery ever.' 'Oh my God, I started my career by making my best ever discovery, so what am I going to do next?', I thought.

I decided to put that aside and move forward. I did what I could. We did the best we could.

It took me a long time to really understand the profound implication of this. I also think it took some time for the community to realise that a new field was really setting up. The public was enthusiastic way more than my colleagues. Fellow physicists were kind of reluctant. They also saw us like a little bit of a threat. 'These people are asking a lot of resources, they want to do a lot of science, but my science is more serious than this kind of exoplanet stuff', they said.

'You are just fashion, it's not going to last guys, it's just trendy', other people told us.

It's interesting to see how things can mature. I know it's a very serious business because you are flying spacecraft on this. I think it took about 10 years for the field to establish itself and to be understood and recognised as a very serious field of physics.

Right now I hope to be successful to move the field to being serious enough and go beyond this to the question of life in the uni- 


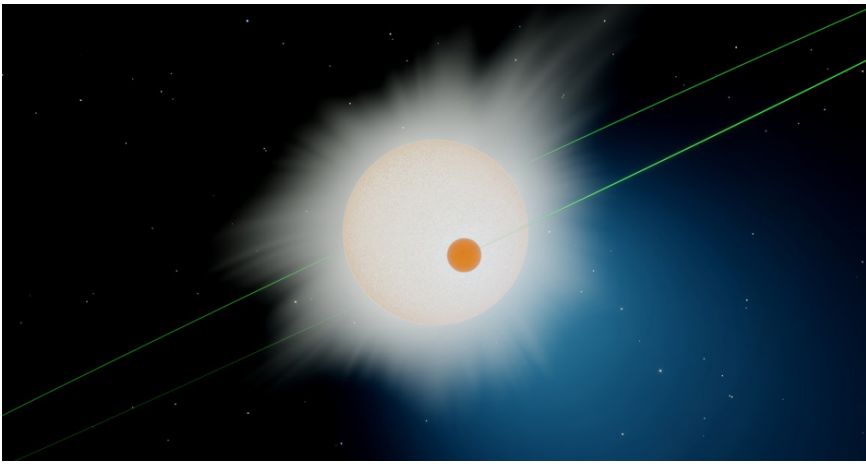

Figure 2 | Artist impression of 51 Peg b orbiting 51 Peg.

verse, which is a very serious field, not only for physics, but also for chemistry and other sciences.

We made a new field of research here. That's why we struggled.

MA. You mentioned that in summer 1994 your PhD advisor (Michel Mayor) gave you the keys of the observatory in south France. What's the story behind that and how did you know what stars you needed to look for?

DQ. This is interesting because it tells a lot about what we were expecting. Nobody was expecting a planet like this one (51 Pegasi b)

The idea was to build the equipment and the equipment was clearly designed to get the right accuracy to find a planet. It was clearly the decision from the beginning to start the program.

My PhD advisor, Michel Mayor, told me 'Start the program, I go in sabbatical, and nothing will happen but start the program. You should be aware that you are starting a long-term program and that you will not be able to continue it.' I said 'fine'.

I built the equipment. I worked on it. I was happy to demonstrate that the equipment was working properly. So, I started the program. We decided to focus on about 100 stars. We selected different stars from the ones that our competitors in Berkeley, Geoffrey Marcy and Paul Butler, were looking at. We knew they were doing a similar study. We knew that it would have been a long race, a long run. So, I really went there and said 'Let's just start and wrap up my $\mathrm{PhD}$ and then I would do something else'.

I initially focussed on the 20 brightest stars out of these 100 . I decided that these stars deserved a bit more measurements than the others, just to check the machine. I took two data points every week. I had one run of observations every two months.

51 Pegasi was one of the stars.

After my first two runs, I came back in January 1995. I kept observing that star and there was something awkward with the data. Data was not stable.

I started to panic here. I said 'Maybe there is something wrong with the data processing of that star'. I really got a bit obsessed about that object. So I kept measuring it to try to understand, try to debug the system up to the point I realised that everything was fine, that my system was working properly. There were no mistakes because it worked well for the other stars. There might have been something special on that star. I looked at that star and I didn't find anything special. It looked like an old star like the Sun.

I concluded that there must have been something orbiting that star and I tried to fit an orbit to the data. But I was so embarrassed. I wanted to come up with a 'real' explanation to Michel. But he was in Hawaii anyway. The communication was very loose because in those days the email was kind of starting.

'I want to go to the end of this, I am going to find this, I am going to get the orbit', I said.

When I finally fitted an orbit I said 'OK, I think I found a planet!'. It's fascinating because I don't think I realised what it really meant at that time. To me it was cool 'I found a planet. Cool, yeah! A 4-days (orbit) planet!'
But I didn't realise it was impossible.

When I mentioned this to Michel, he said, 'Oh... yeah... maybe... let's see'. I think it's obvious he didn't believe me.

He carefully explained 'You know Didier, a planet like that could not exist because the formation theory says...' and told me all the story.

'Oh, I didn't know that', I replied.

I had these very naive perceptions of 'Why not?' In a way, it was a piece of luck because maybe if Michel was there, we might have discarded the observation and said 'Something is wrong and we should not pay attention to that'. So, the fact that I didn't know it was kind of good news because I got focused, obsessed. I got the orbit.

At the end, I explained what I did to Michel, and he had to admit that he had no other explanation and told me 'Look Didier, we are not going to do anything with that. It's so special. We have to leave it as it is, and we have to come back with the telescope. We are going to do a little bit of a game here. We are going to use the textbook of physics. The textbook of physics tells you, when you have a theory, whatever theory it is, the only thing that matters is not how nice is the theory or how revolutionary is the theory or how beautiful is the theory. What matters is, does the theory reflect the data?

You can always find a theory matching data. It's an easy game. You can twist the theory. But it's much more difficult to make a prediction with the theory that would be matched by the data.

You might recall the famous experiment with the solar eclipse of 1919, which demonstrated the validity of Einstein's theory of general relativity. ${ }^{10}$ 'We have to do the same. If this is a planet, your orbit should be valid', Michel said.

'When we come back to the telescope, later on in July, when the star will be back, your prediction should be matched'.

'Okay, let's do that', I said.

In the meanwhile we realised that, because it was a bit edgy, we didn't want to talk too much about that. So we just waited to go back to the telescope in July.

In July we went to the telescope and it was like a dream. You have the theory predicting something and all the data points exactly matching the prediction I made in February.

At that time I think Michel was getting convinced and he said 'Oh my god that's real, so let's write a paper, because it's so easy. The orbiting period is 4 days, maybe Geoffrey Marcy is doing it as well.'

We decided not to talk to anybody apart from our families. We immediately wrote a paper and we submitted it to Nature. The paper was sent to the reviewers to initiate the process.

'I don't believe you, it's an alias', was a comment from one of the referees.

'What can we do? We get more data!' I said.

So we went to the telescope to get more data in September and every day we were collecting data. I think we had two or three data points every night to demonstrate it was not an alias. Then we added the extra data points to the paper and resubmitted it right away.

We had to wait these impossible two months, during which I think Nature didn't know what to do with our paper, because the other referee said 'It's fine'. There were two theoreticians that said 'The theory they mention, migration, maybe it's going to work'. The one dealing with the data said 'I think it's fine, I checked the data and I think this is the only possible orbit, the paper should be published'.

But Nature, delayed and delayed, up to a point where we said 'We are going to announce this to the World, we cannot wait any more'.

'You can do that, but don't say that we've accepted the paper', Nature said.

When we made the announcement in Florence, the world was very sceptical and we were lucky in a sense that our competitors in Berkeley, Geoffrey Marcy and Paul Butler, were observing at a telescope, and somebody told them 'Look, there is a star that the Swiss guy pretends has a planet around, which is just 4 days period, could you have a look at that?'

'Of course, we are going to check it', they said. 
Marcy was a well-known planet-killer in a way that in the past he had already killed lots of claims of exoplanet discoveries.

To me it was fascinating. The only thing I felt was under threat was the data not being confirmed. But one week after, Geoffrey Marcy sent this very nice email. 'Look guys, I confirm everything you said. This is real. Congratulations. You found the first planet'.

To me that was such a relief because I said 'He confirms the data, so everything is fine.' Then we got a bit lucky here because that helped strengthen the facts in the discovery. It turned out not to be enough for the community at large but it was enough for the people that were really interested in looking for the planet.

Nature at that point decided 'Maybe we should accept the paper, because it's too late to stop it'.

But we had been very close to being rejected I think by the editor - I am sure - because it was too big, and we were nobody.

After this some people told us sentences like 'How come NASA is using billions to send spacecraft and they didn't detect the first planet? These are two Swiss guys... somewhere... using a kind of telescope that was built in 1957 in an observatory that was about to close.'

I think this story is very fascinating in terms of sociology. I have just learned recently that our work is part of the ' 10 extraordinary Nature papers. ${ }^{11}$ So now they have changed their mind. We went from being barely accepted to the best 10 .

\section{References}

1 B. Campbell, G. A. H. Walker \& S. Yang. A Search for Substellar Companions to Solar-type Stars. The Astrophysical Journal 331, 902, (1988).

2 A. Wolszczan \& D. A. Frail. A planetary system around the millisecond pulsar PSR1257 + 12. Nature 355, 145-147, (1992).

3 M. Bailes, A. G. Lyne \& S. L. Shemar. A planet orbiting the neutron star PSR1829-10. Nature 352, 311-313, (1991).

4 M. Mayor \& D. Queloz. A Jupiter-mass companion to a solar-type star. Nature 378, 355-359, (1995).

5 D. F. Gray. Absence of a planetary signature in the spectra of the star 51 Pegasi. Nature 385, 795-796, (1997).

6 G. Marcy. Back in focus. Nature 391, 127-127, (1998).

7 D. F. Gray. A planetary companion for 51 Pegasi implied by absence of pulsations in the stellar spectra. Nature 391, 153-154, (1998).

8 A. P. Hatzes, W. D. Cochran \& E. J. Bakker. Further evidence for the planet around 51 Pegasi. Nature 391, 154-156, (1998).

9 E. Cartlidge. Leaders of Faster-Than-Light Experiment Step Down, https:// www.sciencemag.org/news/2012/03/leaders-faster-light-experiment-step-down, (2012).

10 E. Cunningham. Einstein's Relativity Theory of Gravitation. Nature 104, 354 356, (1919).

11 Nature. 10 extraordinary Nature papers, nature.com/collections/fajcgfidgh, (2019).

\section{Conflicts of Interest}

There are no conflicts to declare.

\section{Acknowledgements}

M.A. acknowledges support from the President of the UAE's Distinguished Student Scholarship Program (DSS), granted by the UAE's Ministry of Presidential Affairs. Scientific Video Protocols thanks Dr Subhajit Sarkar and Dr Francesca Faedi for reviewing the manuscript.

\section{Author Contributions}

D.Q. was interviewed by M.A. and M.A. wrote the manuscript.

\section{Keywords}

Breakthrough discoveries, incremental science, exoplanets, 51 Pegasi b.

\section{Article Information}

This article is licensed by Queloz et al. under a Creative Commons Attribution 4.0 International License (CC BY 4.0). 